Remarks on the foregoing plan of Building Earth Walls for Cottages. By J.D.B.

Its adoption will be a great saving in rural economy where lumber and bricks are dear, or cannot be had; and would be preferable to $\log$ cabins in new settlements; such, however, is the force of habit that it will not supersede them; but $I$ have made the above extracts with a view specially to the Liberian Colony, where the timber is not suitable for plank, and lumber for building is mostly imported, and is, of course, very expensive, and stone, or brick walls, equally or more so; and it is of great importance that new settlers, especially farmers, should be cheaply, readily, and comfortably accommodated. These benefits are all united in this plan. It is believed that half a dozen men would, after due preparations, ram up the walls of a cottage eighteen feet square, in two days; and as the natives are already versed in building huts of clay, the change will not be so great to them, but that they might be employed in this mode of building, and probably they would adopt it, as one of the earliest steps toward their civilization.

The only difficulty $I$ apprehend in the execution of the work is, in so adjusting the moulds when drawn up, as to form and preserve the walls truly perpendicular, and with the intended batter, or taper. This will require as correct workmanship as in laying a brick wall. To obviate this difficulty, and put the business within the ability of common workmen, I will propose as a substitute for the moulding board a method that has occurred to me as simple and requiring very little skill, and which may, perhaps, be in other respects preferable-it is this:

The foundation being built, as above directed, of stone or brick, plant posts strongly in the ground on both sides of the foundation, three or four feet apart, in the direction of the walls, and higher than they are to rise. These posts must be in opposite pairs, stiff, and dressed straight on the inner sides, allowing as much space on each side of the foundation as merely to admit the plank, or board, used in the construction, by this mode, to be slipped, or driven, into the spaces. These boards may be of common inch, or inch and quarter, pine, but all used on any one side must be of equal thickness, and all with straight and fair edges and ends. They are to be placed inside of the posts, edge upon edge, as the wall is rammed up, one above another from bottom to top. The plank forming the interior must be cut of lengths to reach exactly from corner, on the outside their ends may project thus-

The posts are secured at top to allow just the space for the thickness of the wall, and of the planks on each side, by tenons made on the posts, over which braces, or gauges, with

above the level, will doubtless be dry, comfortable, and as healthy as brick pavement.

When the walls have become dry, which will require several months, they may be whitewashed with lime, or rough-cast in the usual way, and will then appear as handsome as any stuccoed wall. 
mortices, are placed and secured by wedges on the

$\square$ outer sides. When the wall is made, the wedges being driven out, the gauges, planks, and posts, may be successively removed, and the wall is completed. The only diff. culty I anticipate in this process, will be in planting the posts exactly in true lines. This, however, I think may be effected by first planting the posts nearest to each corner in correct positions; then tixing the braces on them at top and dropping upon them thick plank of such willth as to fill up the space between the posts, which plank will then regulate the tops of all the posts that remain to be set up.

For a single building this plan might be more expensive than the first described, but where a number of houses are to be built, as the same posts and boards will answer for all of them, I believe it would be cheapest, easiest, and certainly best, where good mechanics are wanting.

In putting on the roof it will be prudent to give the eaves a bold projection, to secure the walls from the eflects of rain, and also to put a weather, or wash, board in a sloping position, across the ends of the house (from eve to eve, for the same purpose.

Nothing yet has been said in regard to chimnies. I presume when brick work is easily procured, it may be best to build the chimney therewith when the foundation is laid; but in Liberia, if wanted at all, the fire-place may be built of brick or stone, and the chimney be formed of clay like the native huts, and outside of the wall; similar to the plan pursued with $\log$ houses in this country.

\section{FRANKLIN INSTITUTE.}

Continuation of the Report of the Committee of the Franlin Institute of Pennsylvania, appointed May, 1829, to ascertain, by experiment, the value of Water as a Moving Power.

(Continued from p. 16.)

WHEEL No. IV.

This wheel, six feet in diameter, was substituted for No. III. The barreling of the shaft was removed, and the chain, which supported the weights raised, wound directly upon the axis. 'The iron basket, used in former experiments to contain the weights, was also removed, and the weights were applied directly, by hooks, to the chain.

To ascertain the amount of friction at the gudgeons of the wheel, a cord was wound around the axle, and weights applied until a uniform velocity, of six feet per second, was maintained in the periphery of the wheel, by the descent of the weights. The weight of the wheel was twelve hundred pounds, and seventeen and four-tenths pounds maintained a velocity of six feet per second. The firction was therefore nearly one and a half per cent, (1.45) of the weight. One per cent of the weight applied was, as before, the ratio of friction upon the gudgeons of the drum. 